\title{
Challenges of undertaking a clinical trial using bubble-PEP in an acute exacerbation of chronic obstructive pulmonary disease: A feasibility study.
}

Brigitte Eastwood BPhty, PGCert Physiotherapy (paediatrics)

Physiotherapist, Special Interest Paediatrics, Tauranga Hospital, Bay of Plenty District Health

Nicola Jepsen BHSC (Physiotherapy), PGDipPH

Lecturer, Auckland University of Technology; Physiotherapist, Middlemore Hospital, Counties Manukau Health

Katie Coulter BHSC (Hons) Physiotherapy

Physiotherapist, Sheffield Teaching Hospital, NHS Foundation Trust

Conroy Wong, FRACP

Respiratory Physician, Department of Respiratory Medicine, Middlemore Hospital, Counties Manukau Health

Irene Zeng PhD

Research Biostatistician, Health Intelligence and Informatics, Middlemore Hospital, Counties Manukau Health

\section{ABSTRACT}

The aims of this study were to compare the effect of bubble-positive expiratory pressure with a commercial positive expiratory pressure device and standardised physiotherapy in patients with an acute exacerbation of chronic obstructive pulmonary disease, and to assess the feasibility of inpatient research methods. Patients admitted to a medical ward at Middlemore Hospital, Auckland, New Zealand, with an acute exacerbation of chronic obstructive pulmonary disease were randomly assigned to a treatment group. Participants undertook one supervised treatment, then independently completed two further sessions. Participants' symptoms and ease of sputum expectoration were measured using the Breathlessness, Cough and Sputum Scale. Health-related quality of life and length of hospital stay were also recorded. Only eleven participants were recruited, over ten months. The study was, therefore, underpowered to show differences in end points. Useful findings were uncovered relating to the feasibility of the protocol. Limitations to the recruitment process were identified, including staffing issues and the assessment schedule. Findings from this study will enable revision of the study protocol to allow a modified trial to be performed in the future.

Eastwood B, Jepsen N, Coulter K, Wong C, Zeng I (2016) Challenges of undertaking a clinical trial using bubble-PEP in an acute exacerbation of chronic obstructive pulmonary disease: A feasibility study. New Zealand Journal of Physiotherapy 44(1): 8-16. doi: 10.15619/NZJP/NZJPI44.1.02

Key Words: Pulmonary Disease, Chronic Obstructive; Physiotherapy; Breathing Exercises; Sputum; Cough.

\section{INTRODUCTION}

Chronic obstructive pulmonary disease (COPD) is a respiratory disorder identified by persistent, progressive airflow limitation that is not fully reversible (Global Initiative for Chronic Obstructive Lung Disease 2016, McKenzie et al 2003). Individuals diagnosed with COPD present with chronic, progressive breathlessness, cough, and often sputum production (Seemungal et al 1998). Mucus hypersecretion, ciliary dysfunction, obstruction of airways and loss of elastic recoil of the lungs in COPD impair natural airway clearance (Kim et al 1987, Pryor 1991). Reduced secretion clearance is associated with increased frequency of COPD exacerbations, which are a major cause of hospital admissions and may result in premature death (Maclntyre and Huang 2008). There is limited goodquality evidence for the effectiveness of airway clearance treatments in an acute exacerbation of COPD (AECOPD) (Osadnik et al 2012).

Positive expiratory pressure (PEP) is a form of airway clearance. It splints the airways open on expiration, allowing greater movement of air and more effective sputum movement (Kim et al 1987). In the presence of sputum plugging, PEP allows an increased volume of air to accumulate behind the sputum via collateral ventilation, moving sputum centrally towards larger airways to aid expectoration (Holland and Button 2006). It has been found to be an effective form of airway clearance for people with COPD (Ides et al 2011). Commercial PEP devices are expensive, costing between NZ\$40-160; however, an alternative form of PEP that is inexpensive (costing less than $\$ 2$ to make) and is used by some New Zealand and Australian physiotherapists to manage secretion clearance in COPD, is bubble-PEP (Lee et al 2008, Miller et al 2005). Bubble-PEP is an easily constructed device consisting of a bottle, part-filled with water, and a piece of tubing, through which the patient exhales to create bubbles in the water (Mestriner et al 2009). Despite bubble-PEP being commonly used, there is limited evidence assessing the effectiveness of this particular device for use in secretion clearance in the COPD population (Miller et al 2005). This paper reports a clinician-led feasibility study to investigate the effects of bubble-PEP.

Recruitment problems are common in clinical trials (McDonald et al 2006, Osadnik et al 2012, Seemungal et al 1998, Su et 
al 2007). It is estimated that less than a third of randomised controlled trials successfully achieve their recruitment target in the given time, and $50 \%$ of trials extend recruitment time in order to achieve their target (McDonald et al 2006, Osadnik et al 2012). Clinician-led research brings its own challenges, adding to the complexity of conducting research in the clinical setting. Clinicians undertaking research often lack the time to focus on research, have demanding clinical responsibilities and do not have adequate support staff to carry out a trial (Treweek et al 2013). Many clinician-researchers perceive a conflict between the two roles and do not feel they have adequate training to take on the role of the researcher (Rahman et al 2011). Our study confronted these challenges while undertaking an inpatient clinical trial.

The aims of this feasibility study were firstly, to compare the effect of bubble-PEP with TheraPEP® (a type of commercially available PEP, registered trademark of Smiths Medical) and standardised physiotherapy in patients with an AECOPD, and also to assess satisfaction with the intervention and feasibility of recruitment. This paper reports the methods employed and difficulties encountered when undertaking this clinical trial, including issues around participant recruitment, interventions, assessment scheduling and choice of outcome measures. This discussion may inform the development of a future study.

\section{METHODS}

\section{Design}

This was a single-centre, single-blinded, parallel group trial with adult patients admitted to Middlemore Hospital with an AECOPD with sputum production. The trial received approval from the Health and Disability Ethics Committee (reference number 13/NTA/81) and the Counties Manukau District Health Board Research Committee. It was registered with the Australia New Zealand Clinical Trials Registry, with the Universal Trial Number U1111-1142-1941.
The process for assessment and intervention is outlined in Figure 1. Potential participants were identified via the Ward Information Management System (trademark of PimsProduction) by physiotherapists working Monday to Friday on the medical wards. Participants were assessed by the first researcher, who was blinded to the intervention, within 24 hours of admission. Baseline outcome measures are listed in Figure 1 and further described later in this paper. Participants were randomly allocated to one of three treatment groups - 1) bubble-PEP, 2) TheraPEP® or 3) standardised physiotherapy. Participants received one supervised treatment (supervised by the second researcher) on day one and were reassessed 30 minutes later, using the same outcome measures, by the first researcher. Participants then completed two further independent treatment sessions on day one and completed a diary to document the time and effectiveness of treatment, using outcome measures listed in Figure 1. On day two, participants were reassessed on baseline outcome measures by the first researcher. The second researcher assessed the participant's ability to perform the treatment and collected the diary, and the participant completed the participant satisfaction questionnaire.

\section{Participants}

Participants were recruited between August 2013 and May 2014 from the acute medical wards at Middlemore Hospital. Patients over 18, admitted on week days with an AECOPD and sputum production were considered for inclusion. Inclusion criteria comprised:

- Diagnosis of mild, moderate or severe COPD, based on the Global Initiative for Chronic Obstructive Lung Disease (GOLD) guidelines (2010), with spirometry showing a forced expiratory volume in one second $\left(\mathrm{FEV}_{1}\right) /$ forced vital capacity (FVC) ratio $<0.7$.

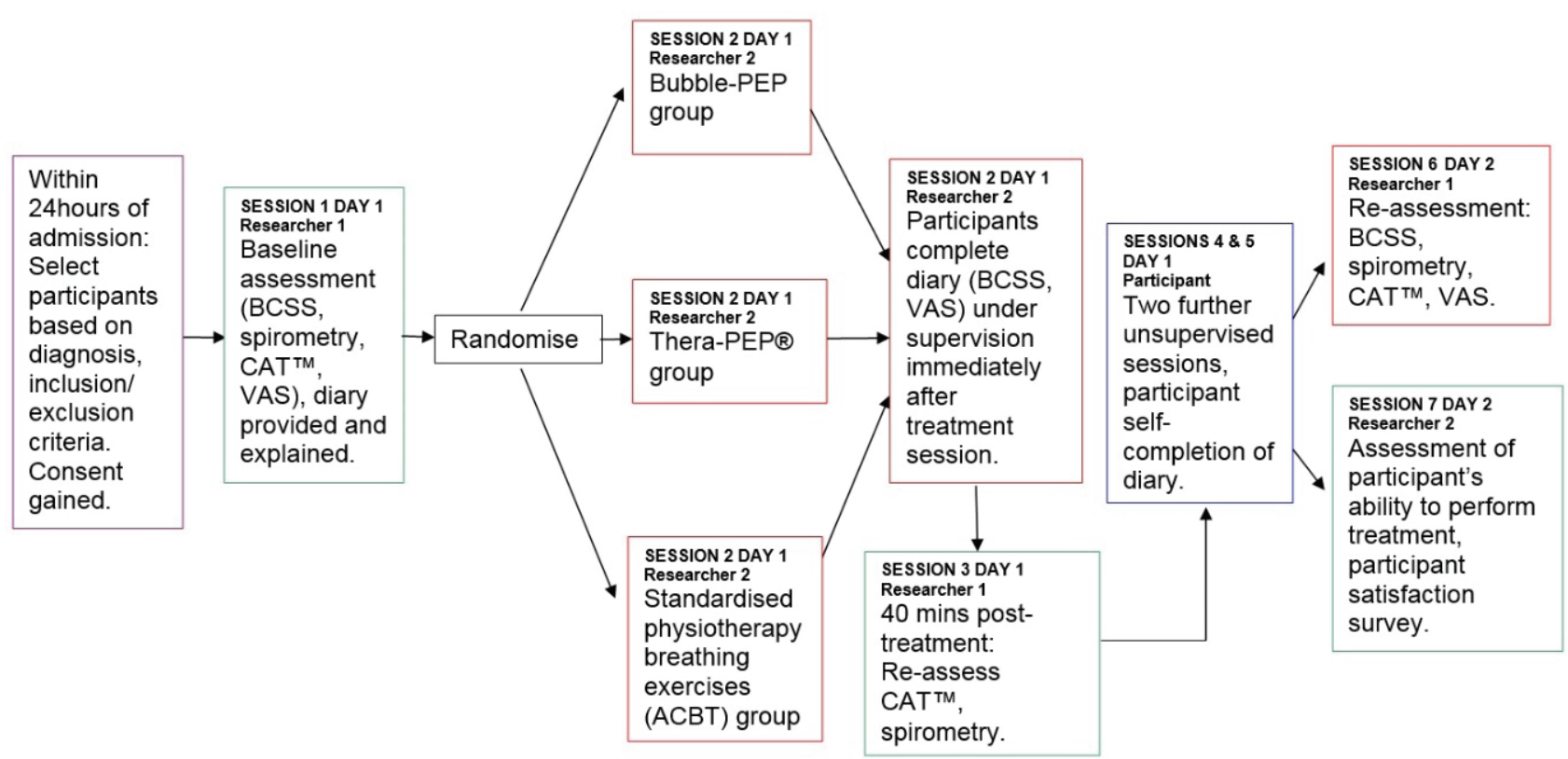

Notes: BCSS, Breathlessness, Cough and Sputum Scale; CAT ${ }^{\mathrm{TM}}$, COPD Assessment Test; VAS, visual analogue scale indicating ease of expectoration; ACBT, Active Cycle of Breathing Technique.

Figure 1: Flow chart showing the process for assessment and intervention 
- Exacerbation with reported sputum, based on GOLD (Global Initiative for Chronic Obstructive Lung Disease 2010) definition: "An event in the natural course of the disease characterised by a change in the patient's baseline dyspnoea, cough and/or sputum that is beyond normal day-to-day variations, is acute in onset, and may warrant a change in regular medication in a patient with underlying COPD" (p 64).

- Able to speak and read English.

- Informed consent to participate in research.

Patients were excluded if they:

- Were unable to complete questionnaires or actively participate in treatment for any other reason.

- Had regularly used any form of PEP at home.
- Were currently receiving bi-level positive airway pressure treatment (as they were already receiving a form of positive pressure).

- Had any contraindications to PEP, for example risk of barotrauma, undrained pneumothorax (American Association for Respiratory Care 1993).

- Had any contraindications to airway clearance techniques, for example active haemoptysis (American Association for Respiratory Care 1993).

- Had been recruited to the trial on a previous admission.

- Had a diagnosis of bronchiectasis, confirmed on high resolution computed tomography, or a highly suspected clinical diagnosis.

The process of recruitment and exclusion is outlined in Figure 2. Eligible participants were randomised using computer-generated

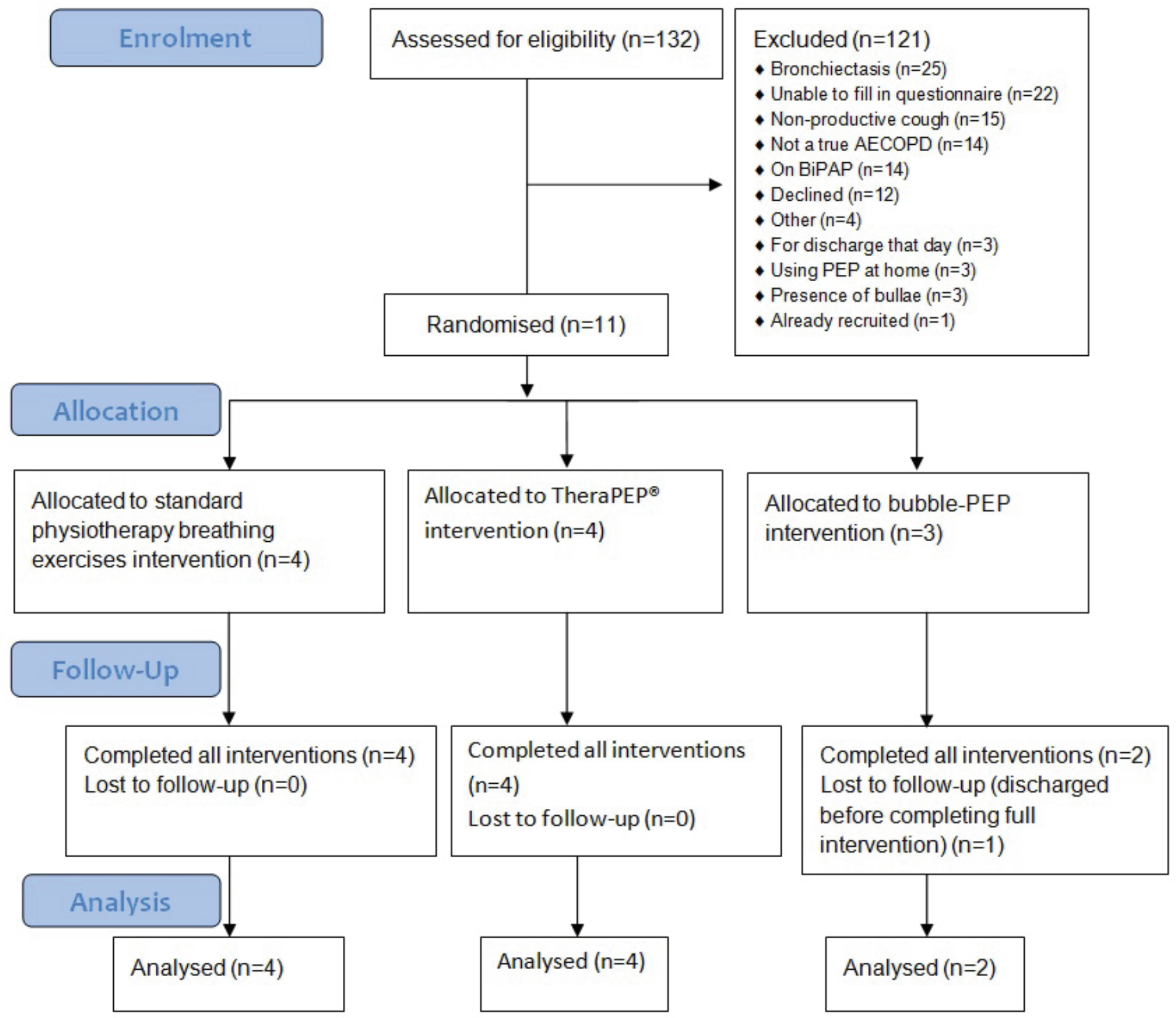

Figure 2. CONSORT diagram (2010) showing the process through the phases of the trial 
treatment codes, which were placed in a sealed envelope and opened prior to the intervention by the second researcher.

\section{Intervention}

Three secretion clearance techniques were carried out with participants in the intervention groups: bubble-PEP, TheraPEP® or the active cycle of breathing technique (ACBT) (Pryor 1991) as the standardised physiotherapy intervention. Each session consisted of three sets of the allocated intervention, with a five minute rest between sets. Participants were provided with a written instruction sheet for their intervention. All groups also received education regarding inhaler use and physical activity, and mobility was assessed as required.

The bubble-PEP intervention was carried out using a homemade bubble-PEP device constructed according to the recommendations outlined by Mestriner et al (2009). The bottle was part-filled with $10 \mathrm{~cm}$ of water to give $10 \mathrm{cmH}_{2} \mathrm{O}$ pressure. A photo of the device is presented in Figure 3. The device was set up in front of the participant on a table, and they were instructed to:

1. Seal their lips around the tubing and exhale with a little force for three seconds to create bubbles.

2. Repeat this for 10 breaths.

3. Perform two huffs (forced expiratory technique demonstration was given).

4. Cough.

Repeat steps 1-4 twice more, with a 5 minute rest in between sets.

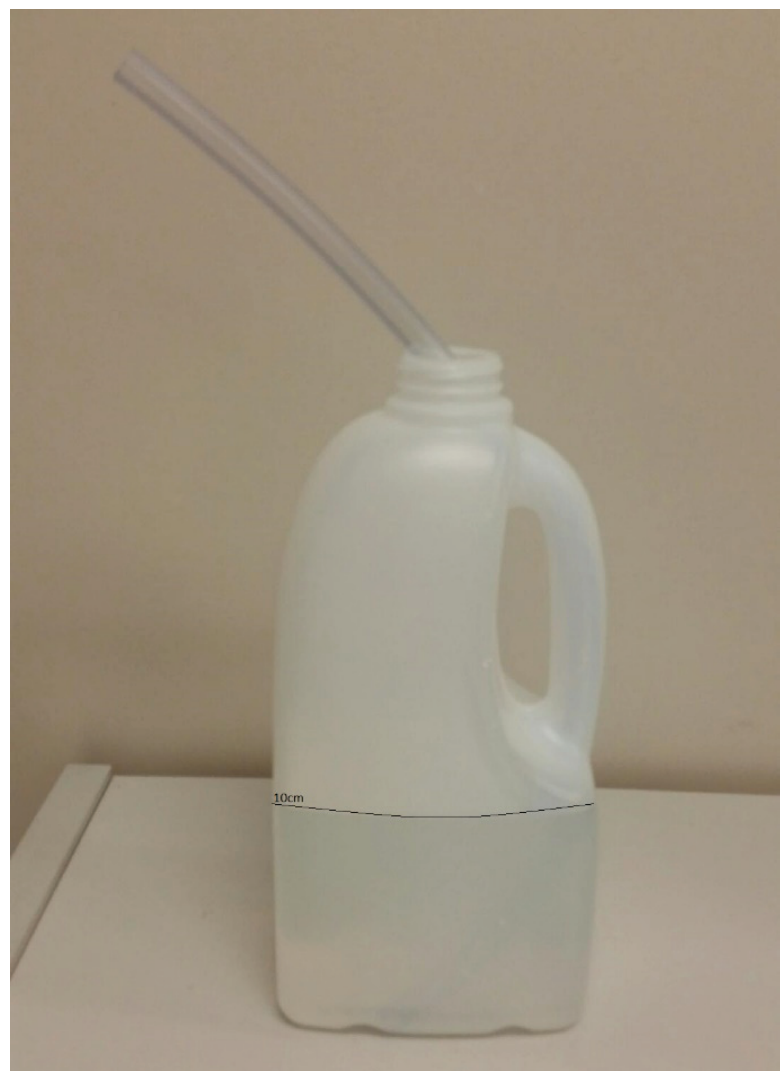

Figure 3. Set-up of bubble-PEP device
The TheraPEP® intervention was carried out as per the manufacturer's instructions, with the participant sitting upright with elbows resting on a table, holding the device in front of them. The participant was instructed to:

1. Seal their lips around the mouth piece and exhale with a little force for three seconds, so the blue disc rises to between the lower and upper black line. Hold at that pressure for a count of three.

2. Repeat this for 10 breaths.

3. If it is too easy to reach the top line, increase the resistance.

4. Perform two huffs (forced expiratory technique demonstration was given).

5. Cough.

6. Repeat steps 1-4 twice more, with a 5 minute rest in between sets.

ACBT, the standardised physiotherapy intervention, was carried out with participants sitting upright. They were instructed to perform:

1. Three slow deep breaths in through their nose.

2. Three relaxed breaths.

3. Three more slow deep breaths.

4. Three relaxed breaths.

5. Two huffs (forced expiratory technique - demonstration was given).

6. One cough.

7. Repeat steps 1-6 twice more, with a 5 minute rest in between sets.

Primary outcome measure

Breathlessness, Cough and Sputum Scale (BCSS)

The BCSS is a valid and reliable outcome measure that is responsive to change in people with COPD (Leidy et al 2003a). While it has not been specifically validated in an inpatient population, it has been shown to identify symptomatic improvements in an AECOPD (Leidy et al 2003a).

\section{Secondary outcome measures}

Length of stay

Length of stay was recorded in hours from the Patient Information Management System (trademark of PimsProduction), from the time of admission until the time the participant left the medical ward.

Visual analogue scale

The visual analogue scale is a $10 \mathrm{~cm}$ horizontal line with 'Very Easy' marked on the left and 'Impossible' on the right. Participants were asked to "please mark on the line how easy you found it to cough up your sputum". The point at which the participant's mark intersects the horizontal line was measured in millimetres from the left of the line.

\section{Spirometry}

Spirometry was completed by one researcher using a Microlab ${ }^{\mathrm{TM}}$ spirometer, registered trademark of CareFusion Corporation. 
The spirometer was calibrated weekly. A standardised instruction sheet, meeting American Thoracic Society and European Respiratory Society standards, was followed (Miller et al 2005). At each assessment session, three good quality blows were completed and the FEV 1 and FVC from the best blow were recorded.

\section{Health-related quality of life - COPD Assessment Test ${ }^{\mathrm{TM}}$ (CATTM)}

The CATTM, registered trademark of GlaxoSmithKline, is a simple, valid and reliable questionnaire for assessing the impact of COPD on quality of life that is strongly correlated with other respiratory-specific quality of life questionnaires (Jones et al 2009). It is recommended for use in clinical trials to assess the ability of interventions to reduce exacerbation severity (Mackay et al 2012).

\section{Participant satisfaction survey}

This survey was created and trialled specifically for use in this study. It contains items on ease of performing the intervention, how effective participants felt their intervention was and reasons for not completing the treatment as prescribed. This gave an indication of compliance and perceived benefits of the three different treatment options.

\section{Ability to perform treatment}

Participants were observed performing the treatment intervention on day two by the second researcher. Participants' ability to perform the treatment was assessed using a visual analogue scale to score their positioning, technique and their ability to follow the written instructions given for the treatment.
This provided useful information for the researchers to assess how easily participants could follow the given instructions.

Sample size

Based on studies by Leidy et al (Leidy et al 2003a, Leidy et al 2003b), the mean (standard deviation) for the BCSS total scores for patients with COPD is reported to be 5.2 (2), and a decrease of greater than 1 point indicates a substantial symptomatic improvement. A power calculation determined that a sample size of 75 (25 in each arm, allowing for a possible $5 \%$ withdrawal rate) would be sufficient to detect a significant difference of 2 points in the BCSS between the active arms and standard care, with a $90 \%$ statistical power.

\section{Statistical analysis}

The continuous variables were presented as either mean and standard deviation, or median and inter-quartile range for the three randomised participant groups. The categorical variables were summarised as frequencies and percentages for each group.

\section{RESULTS}

Approximately 1085 patients were admitted to the hospital with an AECOPD during the 10 month recruitment period (extended from the expected 7 months), including weekend admissions. Of the 132 patients screened, $36(27 \%)$ met the inclusion criteria and $11(8 \%)$ consented to participate in the study. Reasons for exclusion are documented in Figure 2. One participant who was successfully recruited was discharged before undertaking the second day's assessment and treatment sessions and was subsequently lost to follow-up. The other ten participants

Table 1. Participant characteristics by group

\begin{tabular}{|c|c|c|c|c|}
\hline Characteristics & $\begin{array}{l}\text { Standard care } \\
(\mathrm{n}=4)\end{array}$ & $\begin{array}{l}\text { TheraPEP } \\
(n=4)\end{array}$ & $\begin{array}{l}\text { BubblePEP } \\
(n=3)\end{array}$ & $\begin{array}{l}\text { Total } \\
(n=11)\end{array}$ \\
\hline Male & 4 & 3 & 2 & 9 \\
\hline \multicolumn{5}{|l|}{ Ethnicity: } \\
\hline NZ European & 3 & 3 & 2 & 8 \\
\hline Māori & 0 & 1 & 1 & 2 \\
\hline Cook Island Māori & 1 & 0 & 0 & 1 \\
\hline Age (years)* & $73(9)$ & $74(16)$ & $78(11)$ & \\
\hline \multicolumn{5}{|l|}{ Spirometry: * } \\
\hline $\mathrm{FEV}_{1}(\mathrm{~L})$ & $1.1(0.2)$ & $1.0(0.6)$ & $0.8(0.3)$ & \\
\hline $\mathrm{FEV}_{1}(\%)$ & $39.0(7.3)$ & $34.0(13.9)$ & $34.0(11.8)$ & \\
\hline FVC (L) & $2.5(0.92)$ & $2.1(0.95)$ & $1.6(0.15)$ & \\
\hline FVC (\%) & $36(1.2)$ & $37(0.6)$ & $31(0.3)$ & \\
\hline $\mathrm{FEV}_{1} / \mathrm{FVC}(\%)$ & $55.5(8.1)$ & $46.5(13.2)$ & $47.3(17.9)$ & \\
\hline CAT score* $\neq$ & $23.8(2.8)$ & $26.3(6.4)$ & $20.7(15.4)$ & \\
\hline
\end{tabular}

Notes: NZ, New Zealand; FEV forced expiratory volume in one second; FVC, forced vital capacity; CAT, COPD Assessment Test; * Data presented are mean (SD); † Scored from 1-5, where higher scores indicate worse dyspnoea; ₹ Scored from 0-40, where higher scores indicate a greater impact on HRQoL; § Data presented are median (IQR). 
participated in all assessment and treatment sessions. These results provide estimates of eligibility rate $(27 \%)$, decline rate $(33 \%)$ and attrition rate (9\%). Recruitment was ceased after 10 months because of difficulty in recruiting, and researchers leaving the organisation.

Characteristics of participants and groups

A total of eleven participants were recruited. Nine out of eleven participants were male, and most were of New Zealand European ethnicity. COPD severity was similar across groups. All groups had an average $\mathrm{FEV}_{1}$ of between 30 and $50 \%$ predicted, which indicates severe COPD (Global Initiative for Chronic Obstructive Lung Disease 2016). Health-related quality of life scores were similar across groups, as shown by the CATTM. A full outline of participant characteristics is shown in Table 1.

There were no adverse effects experienced by any of the participants during the study. Scores for all outcome measures for each of the three groups are presented in Table 2. On statistical analysis, no significant differences were detected.

Table 2. Mean (SD) scores per group for outcome measures at all re-assessment points

\begin{tabular}{|c|c|c|c|}
\hline Assessment & $\begin{array}{l}\text { Standard Care } \\
(\mathrm{n}=4)\end{array}$ & $\begin{array}{l}\text { TheraPEP } \\
(n=4)\end{array}$ & $\begin{array}{l}\text { Bubble-PEP } \\
(n=3)\end{array}$ \\
\hline \multicolumn{4}{|l|}{ BCSS*: } \\
\hline Day 1, after supervised treatment (30 minutes post-baseline) & $4.3(1.0)$ & $7.0(4.8)$ & $6.5(3.5)$ \\
\hline Day 1 , after unsupervised treatment 1 & $4.8(2.9)$ & $6.0(3.4)$ & $6.5(3.5)$ \\
\hline Day 1 , after unsupervised treatment 2 & $5.3(1.2)$ & $5.5(1.3)$ & $5.0(\mathrm{NA})$ \\
\hline Day 2 re-assessment & $6.8(1.5)$ & $6.0(4.1)$ & $5.0(1.4)$ \\
\hline \multicolumn{4}{|l|}{$\mathrm{VAS}^{+}:$} \\
\hline Day 1 , after supervised treatment & $21.5(19.3)$ & $59(40.8)$ & $57(39.6)$ \\
\hline Day 1 , after unsupervised treatment 1 & $20.3(15.7)$ & $70.8(44.4)$ & $67(39.6)$ \\
\hline Day 1 , after unsupervised treatment 2 & $56.3(40.1)$ & 49 (33.9) & 76 (NA) \\
\hline Day 2 re-assessment & $32.3(34.4)$ & $47.5(44.3)$ & $58(52.3)$ \\
\hline
\end{tabular}

\section{Spirometry:}

Re-assessment day 1 (40 minutes post-treatment)

FEV1

FVC

FEV1/FVC

Re-assessment day 2

FEV1

FVC FEV1/FVC

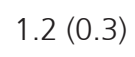

$2.15(0.87)$

$58.3(17.1)$

$1.0(0.1)$

$1.99(0.45)$

$51.5(11.1)$
$1.0(0.5)$

$0.8(0.2)$

$1.92(0.85)$

$1.72(0.27)$

$55.0(16.1)$

$48.0(20.8)$

$0.8(0.3)$

$1.36(0.57)$

$48.5(14.9)$

$1.2(0.7)$

$2.31(1.06)$

$51.0(14.2)$

CAT:

Re-assessment day 1 (40 minutes post-treatment)

$22.5(5.0)$

Re-assessment day 2

$21.3(5.5)$

$24.8(6.9)$

$29.3(3.5)$

$21.8(8.1)$

$27.0(8.5)$

LOS $^{ \pm}$:

$3.5(2.5-7)$

$4(3-5.5)$

$7(1-7)$

Patient satisfaction survey: "The treatment was worthwhile"

\begin{tabular}{llll}
\hline Agree & 1 & 2 & 2 \\
\hline Neutral & 1 & 0 & 0 \\
\hline Disagree & 2 & 2 & 0 \\
\hline
\end{tabular}

Notes: BCSS, Breathlessness, Cough and Sputum Scale; VAS, Visual Analogue Scale; FEV , forced expiratory volume in one second; FVC, forced vital capacity; CAT, COPD Assessment Test; LOS, length of stay; * Scored from 0-12, where higher scores indicate more severe symptoms; † Expressed in millimetres. Participants marked on a $100 \mathrm{~mm}$ line how easy it was for them to clear their sputum, from very easy $(0 \mathrm{~mm})$ to impossible (100mm); $\neq$ Presented as median (IQR). 
Findings of this study show that participants found it easy to perform all treatment interventions. Of the participants who completed the scheduled follow up, all participants in the bubble-PEP group found the treatment worthwhile. In the standardised physiotherapy care group, two participants did not think the treatment was worthwhile and one was neutral about this. In the TheraPEP $®$ group, half of participants thought the treatment was worthwhile and half did not.

\section{DISCUSSION}

Bubble-PEP is widely used by physiotherapy practitioners in New Zealand and Australia as a secretion clearance technique, yet there is little evidence to support its efficacy (Lee et al 2008, Miller et al 2005). Other PEP devices, such as TheraPEP®, have been investigated and found to be useful, but expensive, adjuncts to standard physiotherapy (Ides et al 2011, Su et al 2007). While this study set out to investigate the efficacy of such techniques, several barriers limited recruitment, resulting in a small sample and an insufficiently powered study, hence few conclusions relating to the investigated outcome measures could be made. Lessons were learnt regarding the methods used in this study; these feasibility issues will need to be addressed to enable a fully powered study to be completed.

\section{Feasibility of recruitment}

This study recruited eleven participants over ten months - 1\% of total COPD admissions to the medical wards at Middlemore Hospital over this period, and only $15 \%$ of the recruitment target. Recruitment difficulties are common in clinical trials, for many reasons, including patient eligibility, patients declining to participate and staffing (McDonald et al 2006).

Patient eligibility was a significant problem in the present trial. Many trials find fewer eligible participants than were expected before initiation of the trial (McDonald et al 2006). In this trial, the most common reasons patients were excluded from participating in the study were a comorbid diagnosis of bronchiectasis and being unable to complete questionnaires due to language or other difficulties. Only 12 eligible patients declined to participate. A flow chart indicating reasons participants were excluded is provided in Figure 2.

Inadequate staffing and poor allocation of dedicated research time are common barriers to clinician-led research (Rahman et al 2011). The push for productive patient care often impedes research activity in the clinical setting, and staffing problems, funding limitations and investigators leaving the facility are common reasons that research projects are not completed in Allied Health departments (Bailes and Baldwin 1995). Significant staffing limitations hindered recruitment to this study, which affected the ability to screen and recruit potential participants. Only $12 \%$ of all patients admitted to the hospital with an AECOPD were screened for inclusion. There were four months in which no participants were screened or recruited due to staffing limitations in the wider physiotherapy team, meaning the researchers had to prioritise clinical work over research to ensure adequate patient care. While grant funding was available to fill the clinical roles of the researcher-physiotherapists, their positions were unable to be filled and the clinical physiotherapy team was understaffed during much of the trial period. As well as this, the two lead researchers left the organisation during the extended recruitment period of the study; this was one of the reasons for discontinuing the study. The assessors worked a standard (5 days/week) working week during the time of recruitment. Recruitment was limited to week days to reduce the number of assessors involved and to avoid penal rates for weekend work. Some potentially eligible participants were therefore not recruited as they were admitted over the weekend.

Having a dedicated research team and a longer recruitment period would have ameliorated the above limitations. A research team would be able to approach a greater proportion of patients admitted with an AECOPD, improving the likelihood that an adequate sample could be recruited. Earlier recruitment of participants would also be possible, ensuring that all interventions are undertaken in a timely manner prior to discharge. Researchers working with similar beliefs and attitudes are likely to initiate research and work collaboratively to conduct quality research (Janssen et al 2013). Partnership with a university may be an effective way to conduct clinical research, using an established research team and experienced researchers, who have the resources and skills to assist clinicians to initiate, develop and carry out a research project.

Rahman et al (2011) identify organisational culture as a barrier to clinician-led research. Many clinicians are keen to engage in research, but are not given the time or support from their organisation to do so. Dedicated research teams require adequate funding and support from organisations involved. This must start from within the team - when senior clinicians and managers are involved in research, junior clinicians are likely to follow, helping to build an organisational culture that values research (Janssen et al 2013). Organisations must embrace the potential benefits of clinician-led research to clinicians, patients and the organisation and encourage clinicians to engage in research (Rahman et al 2011).

A multi-centre trial may increase the number of participants eligible for enrolment in the study and would improve the generalisability of findings (Cooley et al 2003, Gul and Ali 2010). Multi-centre trials do, however, have associated disadvantages, such as cost, difficulty maintaining research integrity and cooperation of research teams across sites (Cooley et al 2003). Alternatively, sampling from an outpatient population could aid recruitment of appropriate participants.

Another limitation to recruitment was the reduced sputum load of patients admitted to the medical wards over one winter. Sputum production is not always a clinical feature of COPD - the prevalence of chronic cough and sputum production (chronic bronchitis) has been reported to be between 14 - 35\% in people with COPD (de Oca et al 2012, Kim et al 2011, Lu et al 2010, Munro and Bloor 2010). Patients with a dry cough were not recruited to the study because they did not require therapy for secretion clearance. Expanding the participant group to include those with bronchiectasis would increase the number of potential participants for recruitment.

Feasibility of the intervention

All interventions were performed and tolerated well by 
participants. Of the three techniques, ACBT (standardised physiotherapy) can be taught and performed by people at home free of cost, whereas PEP requires some equipment - bubble-PEP is inexpensive, while TheraPEP $®$ is more costly. In the current economic climate, cost-effective therapy options are important for the maintenance of hospital physiotherapy services. As well as this, for the population involved in this study, cost is a barrier to participation in therapy. For these reasons, ACBT and bubblePEP would be the most feasible options for secretion clearance interventions in hospital and community settings.

Feasibility of the assessment schedule

This study protocol required participants to take part in assessment and treatment on two consecutive days. There were several limitations to this schedule. Firstly, timing three sessions (assessment, treatment and reassessment) on one day was difficult for the assessors, who were also working as clinicians, managing acute case-loads on the medical wards. Secondly, in order for participants to have enough time to complete their assigned treatment three times on the first day, assessments needed to be completed on the morning of that day, adding further pressure to the assessors' scheduling challenges. Lastly, several patients were unable to be recruited as they were preparing for discharge, so would be unable to complete reassessment on day two. Middlemore Hospital has the lowest average length of stay for COPD patients in Australasia; those patients admitted to Middlemore Hospital with an AECOPD during the study's recruitment period had an average length of stay of just 3.6 days. One participant was lost to followup due to being unexpectedly discharged before completing reassessment.

\section{Choice of outcome measures}

Identification of specific, sensitive, valid and reliable outcome measures to assess short-term responses to therapy is a challenge, as demonstrated by this study. There is no goldstandard outcome measure to assess effective secretion clearance. Spirometry was the only objective measure used, which is the internationally accepted tool for diagnosing COPD (McKenzie et al 2003). It is widely used to assess severity of COPD, but is insensitive to sputum transport and the efficiency of secretion clearance techniques (van der Schans 2002).

While the questionnaires used in this study were appropriate, valid and specific outcome measures for identifying symptoms of COPD, the use of questionnaires presented a barrier to recruitment. The Counties Manukau population is multi-cultural; many potentially eligible participants did not speak English and were unable to accurately fill in English questionnaires. Translations of the questionnaires have not been validated, so these patients were excluded from the study. If questionnaires were translated and assessed to have adequate validity, the use of these questionnaires would allow for greater participation from a wider range of the population.

The nature of the inpatient population presents another difficulty with using questionnaires. Those considered for inclusion in the study were predominantly older adults, some of whom reported they found reading and writing challenging due to poor eyesight or dexterity. Some people were therefore excluded because they would not be able to accurately fill in the questionnaires or diary. If the outcome measures were validated to be read out by the researcher then this would also allow for greater participation.

\section{Assessment findings}

This study was not sufficiently powered to detect differences between groups. The sample size required to fulfil the power calculation was 75; this number would only be achievable in a multi-centre trial, or with a dedicated research team who were not juggling clinical duties as well as research.

\section{CONCLUSION}

This study highlighted several unforeseen challenges in the recruitment and assessment process. Undertaking a clinicianled clinical trial in an inpatient population proved difficult, because of the challenges experienced by clinician-researchers in recruiting participants and efficiently carrying out the intervention and assessment. This study suggests that further research investigating the effectiveness of PEP as a secretion clearance technique would be useful, if changes are made to the research protocol. Validation of translated questionnaires will be imperative to aid recruitment. Further investigation of the study population prior to initiation of the trial will also help to set realistic timeframes for recruitment targets.

When undertaking research in the acute medical ward environment, flexibility is required in order to recruit and carry out interventions in a comprehensive and timely manner. It would be more efficient to have a dedicated team of researchers with dedicated research time, who are not simultaneously managing clinical work. Alternatively, a larger group of physiotherapists could each dedicate some time to research, as long as research is prioritised above other competing interests. The challenges faced by clinician-researchers must be recognised; organisations must support and value clinicianled research in order to promote evidence-based health care practice.

\section{KEY POINTS}

1. Bubble-PEP is performed easily and is enjoyed by participants, though there is little firm evidence to support its efficacy.

2. The challenges of managing research and clinical workloads simultaneously limited our ability to efficiently recruit participants to this study.

3. A dedicated research team is necessary to carry out an adequately powered study in the COPD population.

\section{PERIMISSIONS}

This study was approved by the Health and Disability Ethics Committee under the ethics reference code 13/NTA/81. The Universal Trial Number is U1111-1142-1941. Informed consent was obtained from all participants.

\section{DISCLOSURES}

This research was supported by a research grant from the Ko Awatea Maataatupu Emerging Researcher Fund, through Counties Manukau Health. 
The researchers have no conflict of interest to declare.

\section{ADDRESS FOR CORRESPONDENCE}

Brigitte Eastwood, 171 Dickson Road, Papamoa Beach 3118. Telephone: 021257 3099. Email: Brigitte.eastwood@gmail.com

\section{REFERENCES}

American Association for Respiratory Care (1993) AARC clinical practice guideline: Use of positive airway pressure adjuncts to bronchial hygiene therapy. Respiratory Care 38 (5): 516-521.

Bailes AF, Baldwin C (1995) Research in pediatric physical and occupational therapy settings. Physical \& Occupational Therapy in Pediatrics 14 (3/4): 75-93.

CONSORT (2010) The CONSORT flow diagram. http://www.consortstatement.org/consort-statement/flow-diagram [Accessed November 19 2014].

Cooley ME, Sarna L, Brown JK, Williams RD, Chernecky C, Padilla G, Danao $\mathrm{LL}$ (2003) Challenges of recruitment and retention in multisite clinical research. Cancer Nursing 26 (5): 376-384.

de Oca MM, Halbert RJ, Lopez MV, Perez-Padilla R, Tálamo C, Moreno D, Muiño A, Jardim JRB, Valdivia G, Pertuzé J, Menezes AMB (2012) The chronic bronchitis phenotype in subjects with and without COPD: the PLATINO study. The European Respiratory Journal 40 (1): 28-36. doi:10.1183/09031936.00141611.

Global Initiative for Chronic Obstructive Lung Disease (2016) Global strategy for the diagnosis, management and prevention of COPD (updated 2016). http://www.goldcopd.org/ [Accessed 18 January, 2016]

Gul RB, Ali PA (2010) Clinical trials: The challenge of recruitment and retention of participants. Journal of Clinical Nursing 19 (1-2): 227-233. doi:10.1111/j.1365-2702.2009.03041.x.

Holland AE, Button BM (2006) Is there a role for airway clearance techniques in chronic obstructive pulmonary disease? Chronic Respiratory Disease 3 (2): 83-91. doi:10.1191/1479972306cd097rs.

Ides K, Vissers D, De Backer L, Leemans G, De Backer W (2011) Airway clearance in COPD: Need for a breath of fresh air? A systematic review. COPD: Journal of Chronic Obstructive Pulmonary Disease 8 (3): 196-205. doi:10.3109/15412555.2011.560582

Janssen J, Hale L, Mirfin-Veitch B, Harland T (2013) Building the research capacity of clinical physical therapists using a participatory action research approach. Physical Therapy 93 (7): 923-934. doi:10.2522/ptj.20120030.

Jones PW, Harding G, Berry P, Wiklund I, Chen WH, Kline Leidy N (2009) Development and first validation of the COPD Assessment Test. The European Respiratory Journal 34 (3): 648-654. doi:10.1183/09031936.00102509.

Kim CS, Iglesias AJ, Sackner MA (1987) Mucus clearance by two-phase gas-liquid flow mechanism: Asymmetric periodic flow model. Journal of Applied Physiology 62 (3): 959-971

Kim V, Han MK, Vance GB, Make BJ, Newell JD, Hokanson JE, Hersh CP, Stinson D, Silverman EK, Criner GJ (2011) The chronic bronchitic phenotype of COPD: An analysis of the copdgene study. Chest 140 (3): 626-633. doi:10.1378/chest.10-2948.

Lee A, Button B, Denehy L (2008) Current Australian and New Zealand physiotherapy practice in the management of patients with bronchiectasis and chronic obstructive pulmonary disease. New Zealand Journal of Physiotherapy 36 (2): 49-58.

Leidy NK, Rennard SI, Schmier J, Jones MKC, Goldman M (2003a) The breathlessness, cough, and sputum scale: The development of empirically based guidelines for interpretation. Chest 124 (6): 2182-2191. doi:10.1378/chest.124.6.2182

Leidy NK, Schmier JK, Jones MKC, Lloyd J, Rocchiccioli K (2003b) Evaluating symptoms in chronic obstructive pulmonary disease: Validation of the breathlessness, cough and sputum scale. Respiratory Medicine 97, Suppl 1 S59-S70. doi:10.1016/S0954-6111(03)80016-1.
Lu M, Yao W, Zhong N, Zhou Y, Wang C, Chen P, Kang J, Huang S, Chen B, Wang C, Ni D, Wang X, Wang D, Liu S, Lu J, Shen N, Ran P (2010) Chronic obstructive pulmonary disease in the absence of chronic bronchitis in China. Respirology 15 (7): 1072-1078. doi:10.1111/j.14401843.2010.01817.x

Maclntyre N, Huang YC (2008) Acute Exacerbations and Respiratory Failure in Chronic Obstructive Pulmonary Disease. Proceedings of the American Thoracic Society 5 (4): 530-535. doi:10.1513/pats.200707-088ET.

Mackay AJ, Donaldson GC, Patel ARC, Jones PW, Hurst JR, Wedzicha JA (2012) Usefulness of the Chronic Obstructive Pulmonary Disease Assessment Test to evaluate severity of COPD exacerbations. American Journal of Respiratory and Critical Care Medicine 185 (11): 1218-1224. doi:10.1164/rccm.201110-18430C

McDonald AM, Knight RC, Campbell MK, Entwistle VA, Grant AM, Cook JA, Elbourne DR, Francis D, Garcia J, Roberts I, Snowdon C (2006) What influences recruitment to randomised controlled trials? A review of trials funded by two UK funding agencies. Trials 7: 9. doi:10.1186/1745-62157-9.

McKenzie DK, Frith PA, Burdon JGW, Town GI (2003) The COPDX Plan: Australian and New Zealand Guidelines for the management of Chronic Obstructive Pulmonary Disease 2003. The Medical Journal Of Australia 178 Suppl S7-S39.

Mestriner RG, Fernandes RO, Steffen LC, Donadio MVF (2009) Optimum design parameters for a therapist-constructed positive-expiratory-pressure therapy bottle device. Respiratory Care 54 (4): 504-508.

Miller MR, Hankinson J, Brusasco V, Burgos F, Casaburi R, Coates A, Crapo $R$, Enright $P$, van der Grinten CPM, Gustafsson P, Jensen R, Johnson DC Maclntyre N, McKay R, Navajas D, Pedersen OF, Pellegrino R, Viegi G, Wanger J (2005) Standardisation of spirometry. European Respiratory Journal 26 (2): 319-338. doi:10.1183/09031936.05.00034805.

Munro A, Bloor M (2010) Process evaluation: The new miracle ingredient in public health research? Qualitative Research 10 (6): 699-713 doi:10.1177/1468794110380522

Osadnik C, McDonald C, Jones A, Holland A (2012) Airway clearance techniques for chronic obstructive pulmonary disease. Cochrane Database of Systematic Reviews 3. CD008328. doi:10.1002/14651858.CD008328. pub2.

Pryor J (1991) The forced expiration technique. In Pryor J (Ed) Respiratory Care (1st edn). New York: Churchill-Livingstone, pp 79-100.

Rahman S, Majumder MAA, Shaban SF, Rahman N, Ahmed M, Abdulrahman KB, D'Souza UJ (2011) Physician participation in clinical research and trials: Issues and approaches. Advances in Medical Education and Practice 28593. doi:10.2147/AMEP.S14103.

Seemungal TR, Donaldson G, Paul E, Bestall J, Jeffries D, Wedzicha J (1998) Effect of exacerbation on quality of life in patients with chronic obstructive pulmonary disease. American Journal of Respiratory and Critical Care Medicine 157 (5): 1418-1422. doi:10.1164/ajrccm.157.5.9709032.

Su C-L, Chiang L-L, Chiang T-Y, Yu C-T, Kuo H-P, Lin H-C (2007) Domiciliary positive expiratory pressure improves pulmonary function and exercise capacity in patients with chronic obstructive pulmonary disease. Journal of the Formosan Medical Association 106 (3): 204-211. doi:10.1016/S09296646(09)60241-2.

Treweek S, Lockhart P, Pitkethly M, Cook JA, Kjeldstrøm M, Johansen M Taskila TK, Sullivan FM, Wilson S, Jackson C, Jones R, Mitchell ED (2013) Methods to improve recruitment to randomised controlled trials: Cochrane systematic review and meta-analysis. BMJ Open 3 (2). doi:10.1136/ bmjopen-2012-002360

van der Schans CP (2002) Airway clearance: Assessment of techniques. Paediatric Respiratory Reviews 3 (2): 110-114. doi:10.1016/S15260550(02)00004-5. 